OPEN ACCESS

Edited by:

Isabelle Mouyna,

Institut Pasteur, France

Reviewed by:

Irene Castano,

Instituto Potosino de Investigación

Científica y Tecnológica (IPICYT),

Mexico

Paula Sampaio,

University of Minho, Portugal

*Correspondence:

Ali Zarei Mahmoudabadi zarei40@hotmail.com

Specialty section: This article was submitted to Fungi and Their Interactions,

a section of the journal

Frontiers in Microbiology

Received: 02 April 2019 Accepted: 08 July 2019

Published: 31 July 2019

Citation:

Kiasat N, Rezaei-Matehkolaei A and Mahmoudabadi AZ (2019) Microsatellite Typing and Antifungal Susceptibility of Candida glabrata Strains Isolated From Patients With

Candida Vaginitis.

Front. Microbiol. 10:1678. doi: 10.3389/fmicb.2019.01678

\section{Microsatellite Typing and Antifungal Susceptibility of Candida glabrata Strains Isolated From Patients With Candida Vaginitis}

\author{
Neda Kiasat ${ }^{1,2}$, Ali Rezaei-Matehkolaei ${ }^{1,2}$ and Ali Zarei Mahmoudabadi1,2* \\ 1 Infectious and Tropical Diseases Research Center, Health Research Institute, Ahvaz Jundishapur University of Medical \\ Sciences, Ahvaz, Iran, ${ }^{2}$ Department of Medical Mycology, School of Medicine, Ahvaz Jundishapur University of Medical \\ Sciences, Ahvaz, Iran
}

Vulvovaginal candidiasis (VVC) is a yeast infection with a global reach and millions of dollars are spent annually for its diagnosis and treatment. Recently, Candida glabrata with different degrees of antifungal resistance has been considered as the second most common cause of vaginal infections. The aim of the present study is to determine the antifungal susceptibility and molecular epidemiology profiles of $C$. glabrata isolates from patients with WC. Sixty-one C. glabrata isolates were examined for antifungal susceptibility using the EUCAST broth microdilution method. Moreover, microsatellite length polymorphism (MLP) was used for typing the C. glabrata isolates using six microsatellite markers. Overall, 13, 3.3, and $0 \%$ of the isolates were non-wild types to itraconazole, posaconazole, and voriconazole, respectively. Sixty (98.4\%) isolates were an intermediate phenotype to fluconazole and only one isolate was fluconazole resistant. Microsatellite length polymorphism with a discriminatory power of 0.964 identified 35 distinct types and 24 singleton genotypes. The assessment of the population genetic structure revealed that the non-wild-type population had a moderate genetic differentiation compared to the wild type population ( $F_{S T}=0.1457$ ). It was also found that the most common genotypes were G27 (eight strains), G12 (six strains), and G4 (five strains). We found that eight strains were resistant/a non-wild phenotype to itraconazole. Five out of eight (62.5\%) resistant/non-wild phenotype strains correlated to a predominant genotype (GT27) and the rest belonged to GT11 (12.5\%), GT29 (12.5\%), and GT28 (12.5\%). The current study is the first molecular epidemiology study in the southwest of Iran and demonstrates the antifungal susceptibility profiles of $C$. glabrata in it. This study shows a wide range of the genetic diversity of C. glabrata (35 different genotypes) from VVC in the southwest of Iran. The majority of the nonwild isolates had a dominant genotype or genotypes related to this dominant genotype (clonal cluster one).

Keywords: Candida glabrata, vulvovaginal candidiasis, antifungal susceptibility, microsatellite genotyping, Iran 


\section{INTRODUCTION}

Vaginitis or vulvovaginal candidiasis (VVC) is a yeast infection with a global reach and millions of dollars are annually spent for its diagnosis and treatment (Essel et al., 2014). Extensive research has shown that $70-75 \%$ of women have experienced at least one episode of VVC in their lifetime. Furthermore, up to $50 \%$ of the cases have had at least one episode of recurrent vulvovaginal candidiasis (RVVC) (Richter et al., 2005; Amouri et al., 2011; Makanjuola et al., 2018). Although VVC is not a life-threatening infection, it can lead to abortion as well as postpartum and systemic infections (Al-Aali, 2015). Generally, the persistence of RVVC and resistance to antifungals are more common in Candida glabrata (Makanjuola et al., 2018).

The main cause of VVC and RVVC is Candida albicans in more than 80-90\% of cases (Amouri et al., 2011; Sardi et al., 2013; Rezaei-Matehkolaei et al., 2016). Throughout the last decades, the occurrence of VVC with the non-albicans Candida (NAC) species has mainly been due to C. glabrata

TABLE 1 | PCR amplification conditions and contexts each for each Triplex.

\begin{tabular}{|c|c|c|}
\hline Contents & Triplex 1 & Triplex 2 \\
\hline Volume & $25 \mu l$ & $25 \mu l$ \\
\hline $\begin{array}{l}\text { Multiplex TEMpase } 2 x \\
\text { master mix }\end{array}$ & $12.5 \mu \mid$ & $12.5 \mu \mid$ \\
\hline DNA & $3 \mu l$ & $3 \mu l$ \\
\hline $\mathrm{dDW}$ & $3.5 \mu 1$ & $3.5 \mu 1$ \\
\hline \multirow[t]{3}{*}{ Microsatellite markers } & $\begin{array}{l}\text { GLM4, } 5 \text { pmol (Revers, } \\
\text { Forward), } 2 \mu \mathrm{l}\end{array}$ & $\begin{array}{l}\text { RPM2, } 5 \text { pmol (Revers, } \\
\text { Forward), } 2 \mu \mathrm{l}\end{array}$ \\
\hline & $\begin{array}{l}\text { GLM5, } 5 \text { pmol (Revers, } \\
\text { Forward), } 2 \mu \mathrm{l}\end{array}$ & $\begin{array}{l}\text { ERG3, } 10 \text { pmol } \\
\text { (Revers, Forward), } 2 \mu l\end{array}$ \\
\hline & $\begin{array}{l}\text { GLM6, } 5 \text { pmol (Revers, } \\
\text { Forward), } 2 \mu \mathrm{l}\end{array}$ & $\begin{array}{l}\text { MTI, } 10 \text { pmol (Revers, } \\
\text { Forward), } 2 \mu \mathrm{l}\end{array}$ \\
\hline \multirow[t]{5}{*}{ Conditions } & \multicolumn{2}{|c|}{ Denaturation, at $95^{\circ} \mathrm{C}$ for $10 \mathrm{~min}$} \\
\hline & \multicolumn{2}{|c|}{ Denaturation, 30 cycles at $95^{\circ} \mathrm{C}$ for $30 \mathrm{~S}$} \\
\hline & \multicolumn{2}{|c|}{ Annealing, 30 cycles at $55^{\circ} \mathrm{C}$ for $30 \mathrm{~S}$} \\
\hline & \multicolumn{2}{|c|}{ Extension, 30 cycles at $72^{\circ} \mathrm{C}$ for $1 \mathrm{~min}$} \\
\hline & \multicolumn{2}{|c|}{ Final extension, at $72^{\circ} \mathrm{C}$ for $5 \mathrm{~min}$} \\
\hline
\end{tabular}

TABLE 2 | Microsatellite markers.

\begin{tabular}{lll}
\hline Markers & Primers & Primer sequences \\
\hline RPM2 & Forward & FAM 5' ATCTCCCAACTTCTCGTAGCC \\
& Reverse & ACTTGACGACTTGACGCC \\
MTI & Forward & HEX 5' CAGCAATAATAGCTTCTGACTATGAC \\
& Reverse & GACAGGAGCAACCGTTAGGA \\
ERG3 & Forward & TAMRA 5'AGTGCGAGTGTATGTAAAGAATG \\
& Reverse & CGTATACCTTATCTCCGTTCAA \\
GLM4 & Forward & FAM 5' AGTGTTCATTGTCGCCTTC \\
& Reverse & AATGCAGGCTCACCATTTC \\
GLM5 & Forward & HEX 5' TGGGGATAGTGGGAACTCAA \\
& Reverse & CGATGATTCATGTCCGATG \\
GLM6 & Forward & TAMRA 5' GATGATCTGCCCGTTAGGA \\
& Reverse & CCTGAAGTAGGTGCCGAGAG
\end{tabular}

with different degrees of drug resistance and pathogenicity (Sardi et al., 2013; Silva et al., 2017; Segal and Frenkel, 2018). Other NAC species are C. krusei, C. parapsilosis, C. tropicalis, and C. dubliniensis (Richter et al., 2005; Amouri et al., 2011; Al-Aali, 2015; Makanjuola et al., 2018). Generally, NAC species are more frequently isolated from patients with asymptomatic infections and are intrinsically resistant or less susceptible to azoles (Richter et al., 2005; Sardi et al., 2013; Makanjuola et al., 2018).

The epidemiological knowledge of local microorganisms is an important factor in understanding public health problems as well as the treatment and prevention of infectious diseases. In vitro antifungal susceptibility tests and molecular typing are two major keys in epidemiological studies. Several methods have been used for the identification and typing of Candida species including multilocus sequence typing (MLST), matrix assisted laser desorption ionization-time of flight mass spectrometry (MALDITOF MS), random amplification of polymorphic DNA (RAPD), pulsed-field gel electrophoresis (PFGE), multilocus enzyme electrophoresis (MLEE), and fingerprinting with complex DNA probes (Dodgson et al., 2003; Dhieb et al., 2015; Amanloo et al., 2017). However, microsatellite analysis based on short tandem repeats (STR's) has been considered as a rapid and reliable technique with a highly discriminatory power (DP) for typing of C. glabrata (Abbes et al., 2012; Hou et al., 2017).

Vulvovaginal candidiasis is one of the most common fungal infections among middle-aged Iranian women (Mahmoudabadi et al., 2010; Rad et al., 2011; Mohammadi-Ghalehbin et al., 2016; Rezaei-Matehkolaei et al., 2016). Several frequencies of the disease have been reported from different provinces in Iran including 62.1, 43.3, 40, 26, and 4.8\% from Ilam, Babol, Arak, Hamadan, and Zanjan, respectively (Jamilian et al., 2007; Bahram et al., 2009; Esmaeilzadeh et al., 2009; Mohamadi et al., 2015; Habibipour, 2016).

Considering the above points, this study aims to investigate the genotyping of C. glabrata strains isolated from VVC in the southwest of Iran based on microsatellite markers. Moreover,

TABLE 3 | Demographic characteristics of patients with vulvovaginal candidiasis.

Vaginitis (61)

Age range

18-56 year

Sample sources

Ahvaz

Bushehr

$46(75.4 \%)$

Unknown

$10(16.4 \%)$

Vaginitis type

Single episode or acute WC $30(49.2 \%)$

Multiple episode $\quad 23(37.7 \%)$

Unknown

$8(13.1 \%)$

Predisposing factors

Low-dose estrogen (LD) user 15 (65.2)

$\begin{array}{ll}\mathrm{HIV}^{+} & 5(21.7)\end{array}$

Pregnant $3(13.1)$

Non $\quad 38(62.3 \%)$ 
the most common genotypes and their association with azoles susceptibility are also investigated.

\section{MATERIALS AND METHODS}

\section{Isolates and Identification}

In the present study, 61 clinical C. glabrata strains from patients with VVC were collected. Forty-six (75.4\%) strains were isolated from the vaginal samples of patients in Ahvaz (from January 2017 to March 2018), five (8.2\%) strains were unknown (related to cities around Ahvaz), and 10 (16.4\%) strains were isolated from the vaginal samples of patients in Bushehr city. These isolates were initially identified using the morphological and microscopic features including small yeasts with budding cells without hyphae/pseudohyphae on corn meal agar (Difco, United States) with $1 \%$ Tween 80 and its pink colored colonies on CHROMagar ${ }^{\mathrm{TM}}$ Candida (CHROMagar, Paris, France). Then, the isolates were confirmed by PCR using ITS1/ITS4 primers and the PCR products were subjected to sequence analysis (White et al., 1990). All the sequences were compared to reference sequences in the GenBank (NCBI) database via the nucleotide BLAST $^{\mathrm{TM}}$ algorithm (similarity values $\geq 99 \%$ ). Finally, all the nucleotide sequences were recorded in the GenBank database.

\section{Microsatellite Analysis}

The genomic DNA was extracted from the overnight cultures using a lysis buffer and boiling method, purified by phenolchloroform-isoamyl alcohol (Sigma-Aldrich, Germany), precipitated with isopropanol (Merck, Germany), and washed with $70 \%$ ethanol. The DNA was then dried in room temperature and preserved at $-20^{\circ} \mathrm{C}$. Microsatellite length polymorphism (MLP) was performed using two separate triplex PCRs ([GLM4, GLM5, and GLM6] and [RPM2, ERG3, and MTI]) (Table 1) (Abbes et al., 2011, 2012).

Six polymorphic microsatellite markers including RPM2, ERG3, MTI, GLM4, GLM5, and GLM6 were used for microsatellite typing (Abbes et al., 2011; Hou et al., 2017). Each forward primer was labeled at the $5^{\prime}$-side with one of the FAM, HEX, and TAMRA fluorophores (Table 2). Amplification reactions and their programs were performed as described by Abbes et al. (2012). The PCR products were sent to the Macrogen Company for fragment analysis by capillary electrophoresis on an ABI3730XL DNA Analyzer (Applied Biosystems). The internal control used by the capillary electrophoresis was the Genescan $^{\text {TM }} 500 \mathrm{Liz}^{\circledR}$ size standard. Finally, the lengths of the alleles were measured with the GeneMapper Software 5 (Applied Biosystems).

\section{Antifungal Susceptibility Assay}

The in vitro antifungal susceptibility of 61 C. glabrata isolates to four azole drugs (fluconazole $[32 \mathrm{mg} / \mathrm{ml}$ ] [Serva, United States], itraconazole $[2.5 \mathrm{mg} / \mathrm{ml}]$ [Sigma-Aldrich, Germany], posaconazole $[1.75 \mathrm{mg} / \mathrm{ml}]$ [Sigma-Aldrich, Germany], and voriconazole [1.25 $\mathrm{mg} / \mathrm{ml}$ ] [Sigma-Aldrich, Germany]) was determined using the European Committee on Antimicrobial Susceptibility (EUCAST) broth microdilution

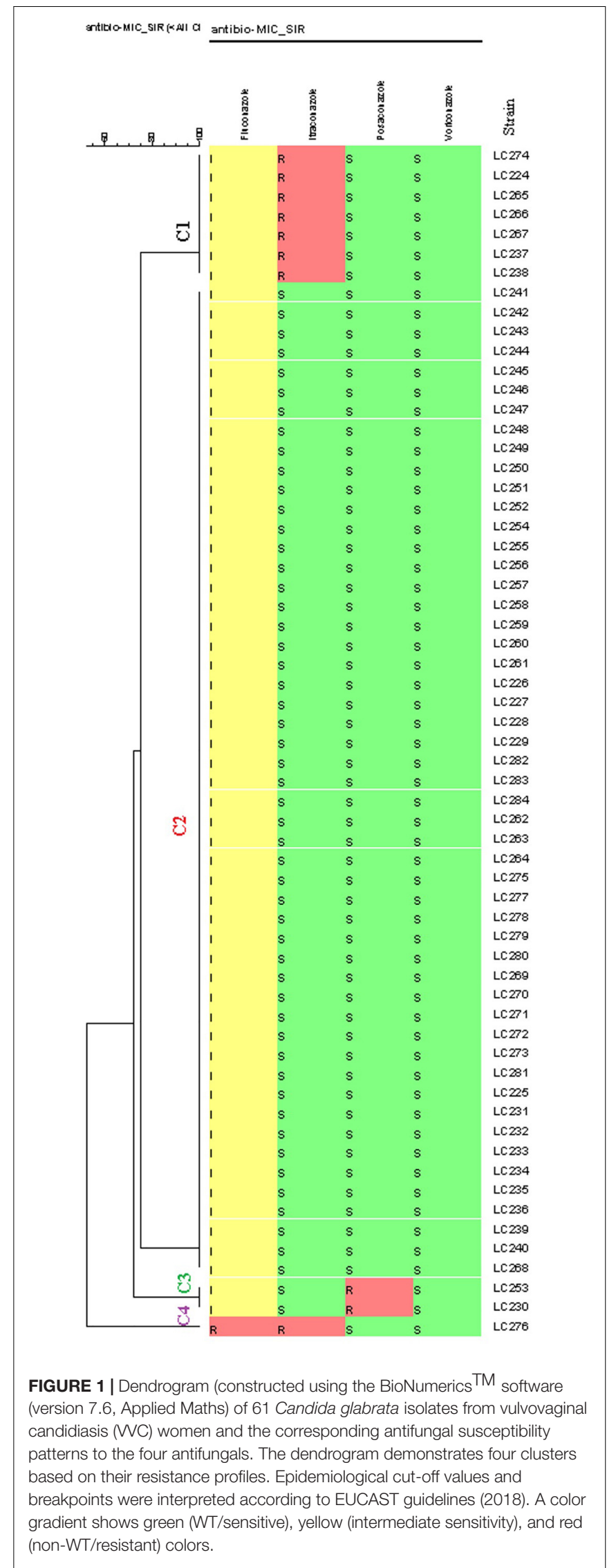




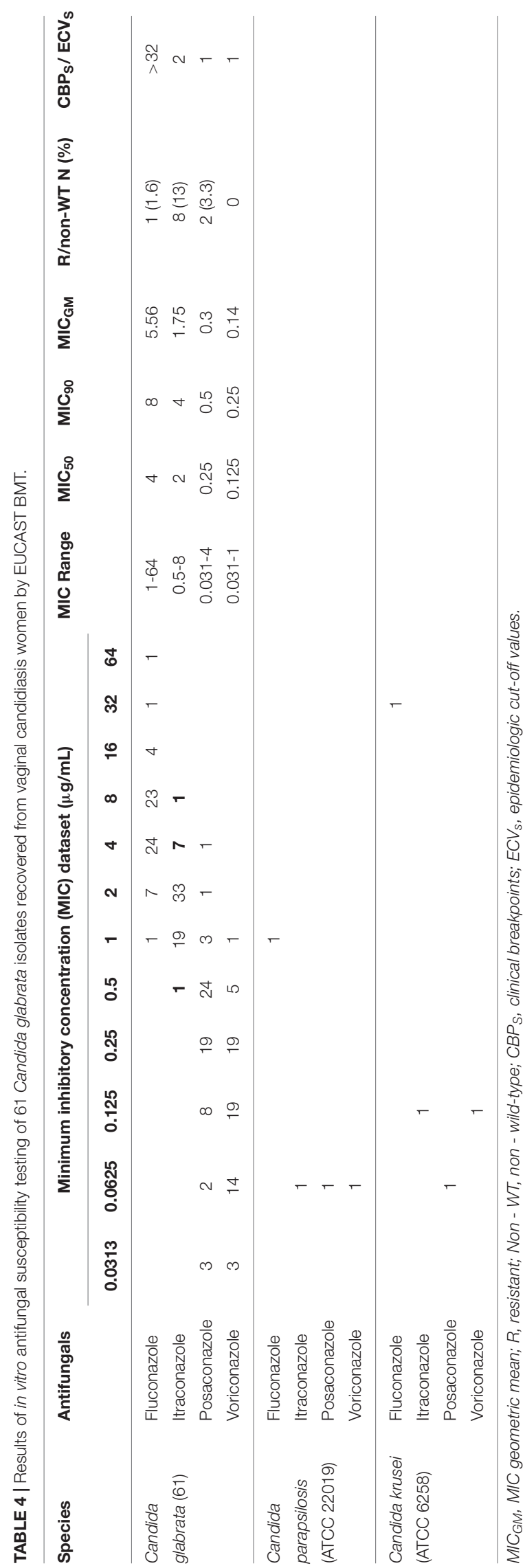

method [EUCAST definitive document EDef 7.3.1 revision 2017 EUCAST (2017)]. This method was modified with colorimetric indicator Resazurin (Gharaghani et al., 2016) to read the MIC more easily. The MIC is defined as the lowest concentration of an antifungal drug that inhibits the fungal growth. $\mathrm{MIC}_{50}$ and $\mathrm{MIC}_{90}$ were determined as the lowest concentrations of the antifungal drug inhibiting 50 and $90 \%$ of the yeast growth, respectively. According to the EUCAST antifungal clinical breakpoint (v.9.0 valid from 2018-02-12) and Klotz et al. (2016), the fluconazole MIC ratios of $>32,>0.002-32.0$, and $\leq 0.002 \mu \mathrm{g} / \mathrm{ml}$ were interpreted as resistant, intermediate, and susceptible.

There are no clinical breakpoints for itraconazole, posaconazole, and voriconazole of C. glabrata isolates in the EUCAST broth microdilution test (BMT) guidelines. Therefore, species-specific epidemiological cut-off values (ECVs) were used for the interpretation of isolates as a wild-type (WT) or a non-WT. The ECV for the non-WT to voriconazole and posaconazole is MIC $>1 \mu \mathrm{g} / \mathrm{ml}$ while for itraconazole it is MIC $>2 \mu \mathrm{g} / \mathrm{ml}$ (Meletiadis et al., 2017). Furthermore, MIC ${ }_{50}$, $\mathrm{MIC}_{90}$, and $\mathrm{MIC}_{\mathrm{GM}}$ (geometricmean) were calculated for each antifungal drug. The $\mathrm{MIC}_{\mathrm{GM}}$ is an average or mean which shows the central tendency or typical value of a set of MICs. In this study, $\mathrm{MIC}_{\mathrm{GM}}$ was calculated using online software ${ }^{1}$. Candida parapsilosis ATCC 22019 and Candida krusei ATCC 6258 were also used as quality control organisms for the antifungal assay.

\section{Statistical Analysis}

The dendrogram and minimum spanning tree (MSTree) algorithms were constructed with BioNumerics ${ }^{\mathrm{TM}}$ software (version 7.6, Applied Maths, License period: valid from 11/October/2018 until 10/November/2018; License string: 2KCN-45RP-DND7-47WW-FVHP-UV2M) using a categorical value to define the genetic relatedness between the C. glabrata isolates. The DP was calculated based on Simpson's index of diversity $^{2}$ (Chillemi et al., 2016).

The Wright's Fixation Index $\left(\mathrm{F}_{\mathrm{ST}}\right)$ was measured by the FSTAT software version $2.9 .3^{3}$ for the interpretation of genetic differentiation among the populations of $C$. glabrata isolates. The $\mathrm{F}_{\mathrm{ST}}$ values of $0.0-0.05,0.05-0.15,0.15-0.25$ and higher than 0.25 demonstrate little, moderate, great and fully genetic differentiations, respectively (Abbes et al., 2015).

\section{RESULTS}

\section{Demographic Characteristics}

Of the 61 VVC patients with C. glabrata, $49.2 \%$ had a single episode or acute VVC, $37.7 \%$ had two or more than two episodes of VVC in the last year (multiple episodes), and $13.1 \%$ had an unknown history. The demographic details of the patients are shown in Table 3.

\footnotetext{
${ }^{1}$ http://www.meracalculator.com/math/geometric-mean.php

${ }^{2}$ http://insilico.ehu.es/mini_tools/discriminatory_power/index.php

${ }^{3}$ http://www2.unil.ch/popgen/softwares/Fstat293dist.exe
} 
TABLE 5 | Features of six microsatellite markers for the 61 Candida glabrata isolates from Candida vaginitis patients.

\begin{tabular}{|c|c|c|c|c|c|c|}
\hline Markers & RPM2 & MTI & ERG3 & GLM4 & GLM5 & GLM6 \\
\hline Number of alleles & 5 & 9 & 12 & 14 & 8 & 10 \\
\hline Range size (bp) & $116-140$ & $227-241$ & $184-353$ & $134-326$ & $254-300$ & $260-329$ \\
\hline Diversity index & 0.710 & 0.827 & 0.807 & 0.871 & 0.733 & 0.809 \\
\hline
\end{tabular}

\section{Identification of the Isolates}

The DNA sequencing analysis of 61 isolates was confirmed as C. glabrata and recorded on NCBI GenBank (Accession No. LC389224-84).

\section{Antifungal Susceptibility Testing (AFST)}

According to the EUCAST BMT assay and the generated dendrogram, C. glabrata isolates were divided into four clusters (C): Cluster 1 (C1) containing seven isolates with a non-WT phenotype to itraconazole, Cluster 2 (C2) containing isolates that were neither a non-WT phenotype nor a resistant phenotype to the tested antifungals, Cluster 3 (C3) containing two isolates with a non- WT phenotype to posaconazole, and Cluster 4 (C4) containing one isolate with a resistant phenotype to fluconazole and a non- WT phenotype to itraconazole. Therefore, only one isolate $(1.6 \%)$ was cross-resistant/a non-WT to fluconazole and itraconazole in this study (Figure 1).

Sixty (98.4\%) of the 61 isolates of C. glabrata were intermediate to fluconazole and one (1.6\%) was resistant to fluconazole. The rates of non-WT to itraconazole and posaconazole were 13 and $3.3 \%$ of the C. glabrata isolates, respectively. One hundred percent of C. glabrata isolates had WT MICs to voriconazole (Table 4). In general, in this study, $10(16.4 \%)$ of the isolates were resistant/a non-WT to all the tested antifungals. Seven of them were a nonWT to itraconazole, two isolates were a non-WT to posaconazole, and one isolate was both resistant to fluconazole and a non-WT to itraconazole.

\section{Microsatellite Analysis}

In the present study, 58 different alleles were identified using six microsatellite loci (RPM2, MTI, ERG3, GLM4, GLM5, and GLM6) in 61 C. glabrata isolates. The DP is the average probability that the typing system shows for different types of two unrelated strains randomly sampled in the microbial population of a given taxon. The DP range is from 0 to 1 and the highest DP $(\mathrm{DP}=1)$ indicates that the typing system discriminates between all the isolates. The highest and lowest genotypic diversities were attributed to locus GLM4 $\left(\mathrm{DP}_{\text {value }}=0.871\right)$ and locus RPM2 $\left(\mathrm{DP}_{\text {value }}=0.710\right)$ (Table 5). Moreover, 35 different genotypes $(\mathrm{DP}=0.964)$ were obtained from 61 unrelated $C$. glabrata isolates with 24 singleton genotypes (Figure 2). Accordingly, the most frequent genotype was GT27 (eight strains, 13.1\%) followed by genotypes GT12 (six strains, 9.8\%) and GT4 (five strains, 8.2\%). The other remaining genotypes had a frequency of below $5 \%$.

\section{Genotype and Susceptibility to Antifungals}

The association between the microsatellite genotype and antifungal drug resistance profile of C. glabrata isolates is shown by the minimum spanning tree (MSTree). Based on MSTree (Figure 3), one isolate of the genotype GT1 and the only isolate of genotype GT31 were a non-WT to posaconazole. One isolate of genotypes GT28, GT29, and GT11 and five isolates of genotype GT27 were a non-WT to itraconazole. Also, only one C. glabrata isolate was associated with cross-resistant/a nonWT to itraconazole and fluconazole and had genotype GT27. In total, it was found that $50 \%(5 / 10)$ of resistant/non-WT isolates to antifungals belonged to the most common genotype (GT27). In other words, $62.5 \%$ (five of eight) of resistant/non-wild phenotype strains to itraconazole correlated to the predominant genotype (GT27) and the rest belonged to GT11 (12.5\%), GT29 (12.5\%), and GT28 (12.5\%). Hence, the majority of resistant C. glabrata isolates to antifungal drugs, especially itraconazole, had a dominant genotype, GT27, or genotypes related to this dominant genotype (clonal cluster one).

\section{Population Genetic Analysis}

Wright's $\mathrm{F}_{\mathrm{ST}}$ is a measure of the population substructure and is used to analyze the genetic structure differences among populations. $\mathrm{F}_{\mathrm{ST}}$ value was evaluated between the single-episode and multiple-episode populations $\left(\mathrm{F}_{\mathrm{ST}}=0.0053\right)$. This finding demonstrated a small genetic structure between two genetic groups of C. glabrata isolates. Moreover, $\mathrm{F}_{\mathrm{ST}}$ was calculated for the non-WT and WT populations $\left(\mathrm{F}_{\mathrm{ST}}=0.1457\right)$. The wild-type population had a moderate genetic diversity compared to the non-WT population (Table 6).

\section{Association of Iranian Genotypes With Those of Other Countries}

Figure 4 shows the MSTree association of the microsatellite genotypes of Iranian C. glabrata isolates with those of other countries that used similar microsatellite markers. Based on the MSTree, Iranian genotypes of C. glabrata isolates are fully distinct from those in other countries (Abbes et al., 2012; Chillemi et al., 2016; Hou et al., 2017).

\section{DISCUSSION}

Based on the WT defined by the EUCAST method for speciesspecific C. glabrata, only $16.4 \%$ (10 of 61) of C. glabrata isolates were resistant $(\mathrm{r}) /$ had reduced susceptibility (non-WT) to azoles (itraconazole, voriconazole, posaconazole, and fluconazole). In our results, resistance $(16.4 \%)$ was about $10 \%$ more than that obtained by Hou et al. (2017) (6.8\%) who tested the isolates with miscellaneous sources. Moreover, by employing EUCAST breakpoints and epidemiological cut-off values, Klotz et al. (2016) 


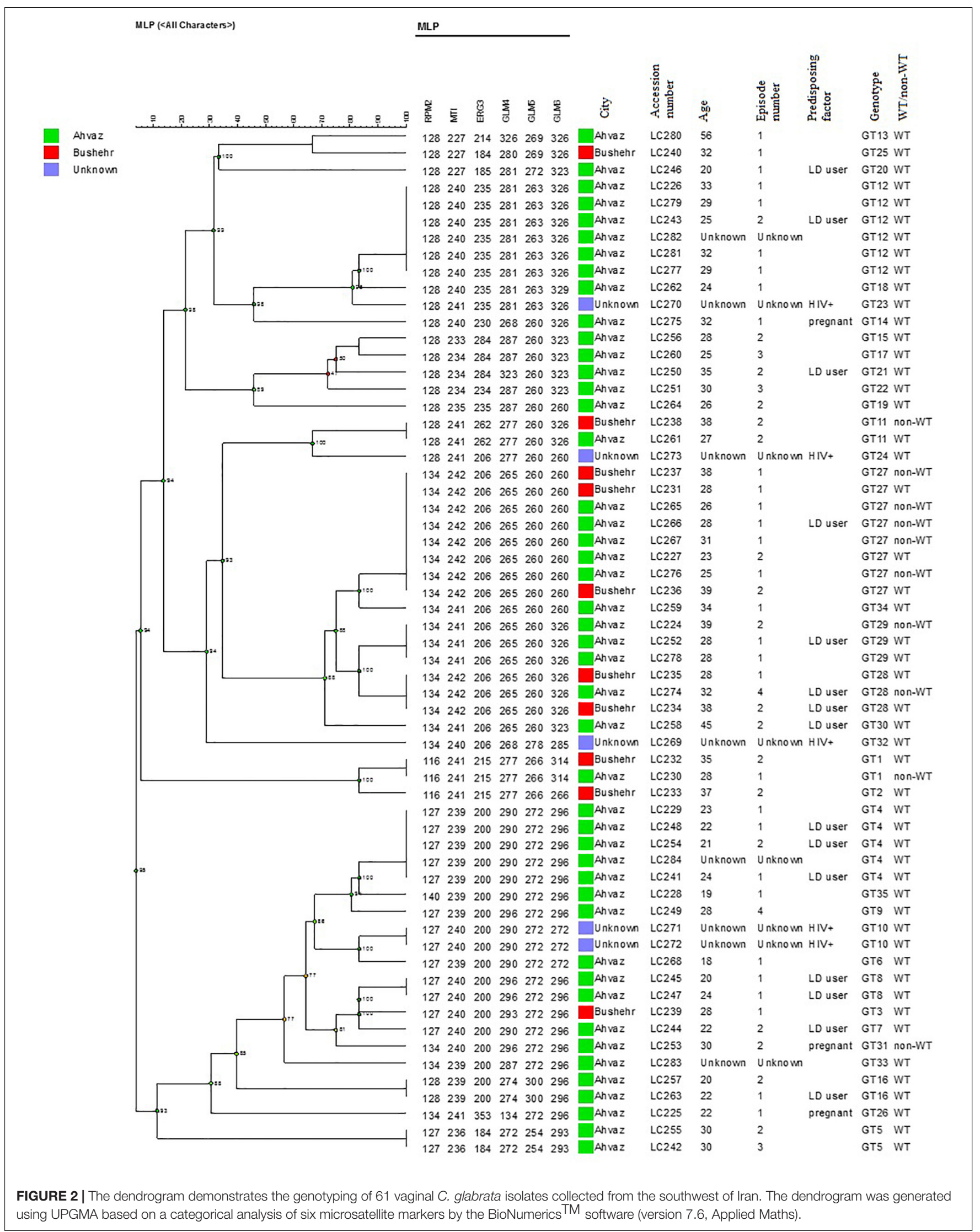




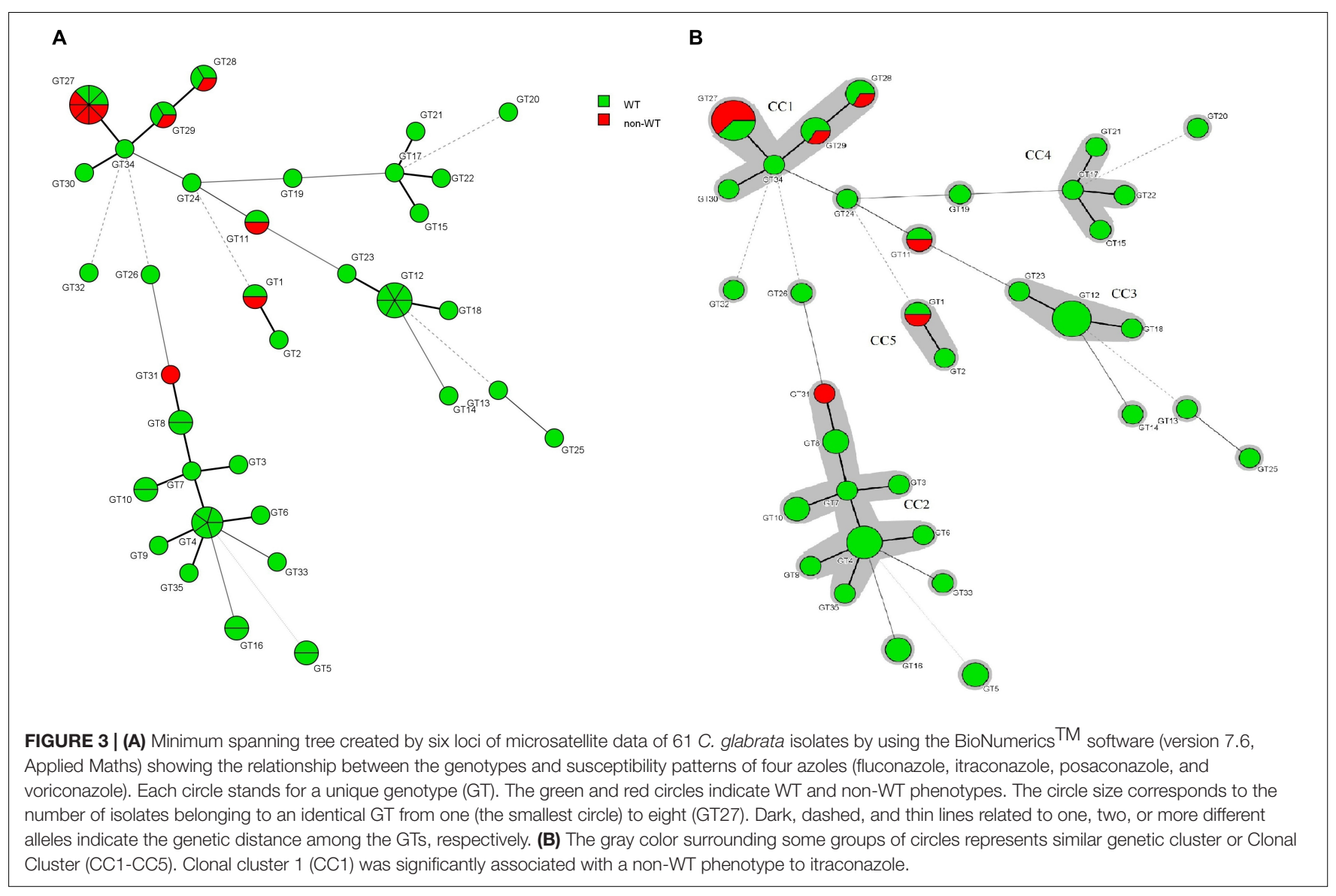

mentioned that 25 and $30 \%$ of the blood isolates were r/a nonWT to fluconazole and voriconazole, respectively. It seems that the difference among the isolates resistant to azoles can be due to the site of isolation. On the other hand, all the C. glabrata isolates were susceptible to voriconazole and only one isolate was resistant to fluconazole. These results are comparable to those of Meletiadis et al. (2017). They demonstrated that the resistance rates to C. glabrata were $2.3 \%$ for fluconazole and $1.7 \%$ for voriconazole. We did not observe any isolate of C. glabrata that was susceptible to fluconazole and 98.4 and $1.6 \%$ of them were intermediate and resistant to fluconazole, respectively. This trend in reduced susceptibility to fluconazole is a warning for C. glabrata isolates, which have acquired a significant resistance to it. Klotz et al. (2016) and Meletiadis et al. (2017) have found that none of the C. glabrata isolates were susceptible to fluconazole which was similar to our results.

Several studies have reported high resistance rates of C. glabrata isolates to itraconazole with different percentages. Our results revealed that the highest resistance rate $(13 \%)$ was associated with itraconazole, which is consistent with the studies of Shokohi et al. (2011) (12.5\%) and Espinel-Ingroff et al. (2005) (17\%). On the other hand, our results are inconsistent with those of Richter et al. (2005) and Amirrajab et al. (2016) who observed a resistance of up to $70 \%$ to itraconazole. This difference may be due to the difference in the source of samples and less prescription of antifungal drugs for the treatment of vaginal
TABLE 6 | Wright's $F_{S T}$ values for subdivided populations of Candida glabrata isolates.

\begin{tabular}{llcc}
\hline Populations & Isolates (No.) & F \\
\hline A & $\begin{array}{l}\text { single-episode }(n=30) \text { vs. } \\
\text { multiple-episode }(n=23)\end{array}$ & 53 & 0.0053 \\
B & $\begin{array}{l}\text { WT }(n=51) \text { vs. non-WT } \\
(n=10)\end{array}$ & 61 & 0.1457 \\
\end{tabular}

FST values calculated by FSTAT software version 2.9.3; Population genetic analysis between Candida glabrata isolates with different episode (A) and between the wild type and non-wild type phenotype for fluconazole, itraconazole, posaconazole, and voriconazole (B).

infections. In other words, resistance to itraconazole correlates with a prior history of the use of itraconazole for prophylaxis and therapy. A lower percentage of resistance to itraconazole (1.2\%) was reported by Meletiadis et al. (2017). In our study, the rate of posaconazole resistance $(3.3 \%)$ was nearly the same as that of Meletiadis et al. (2017) (2.3\%).

In this study, we studied the genetic diversity and population structure of C. glabrata isolates collected from the southwest of Iran. Notably, all the loci demonstrated a degree of allelic variation from five to 14 alleles for RPM2 and GLM4 markers, respectively. Most of the alleles were common among C. glabrata isolates, whereas some of them were less frequent or unique and rare. Finally, our results demonstrated 35 different genotypes 


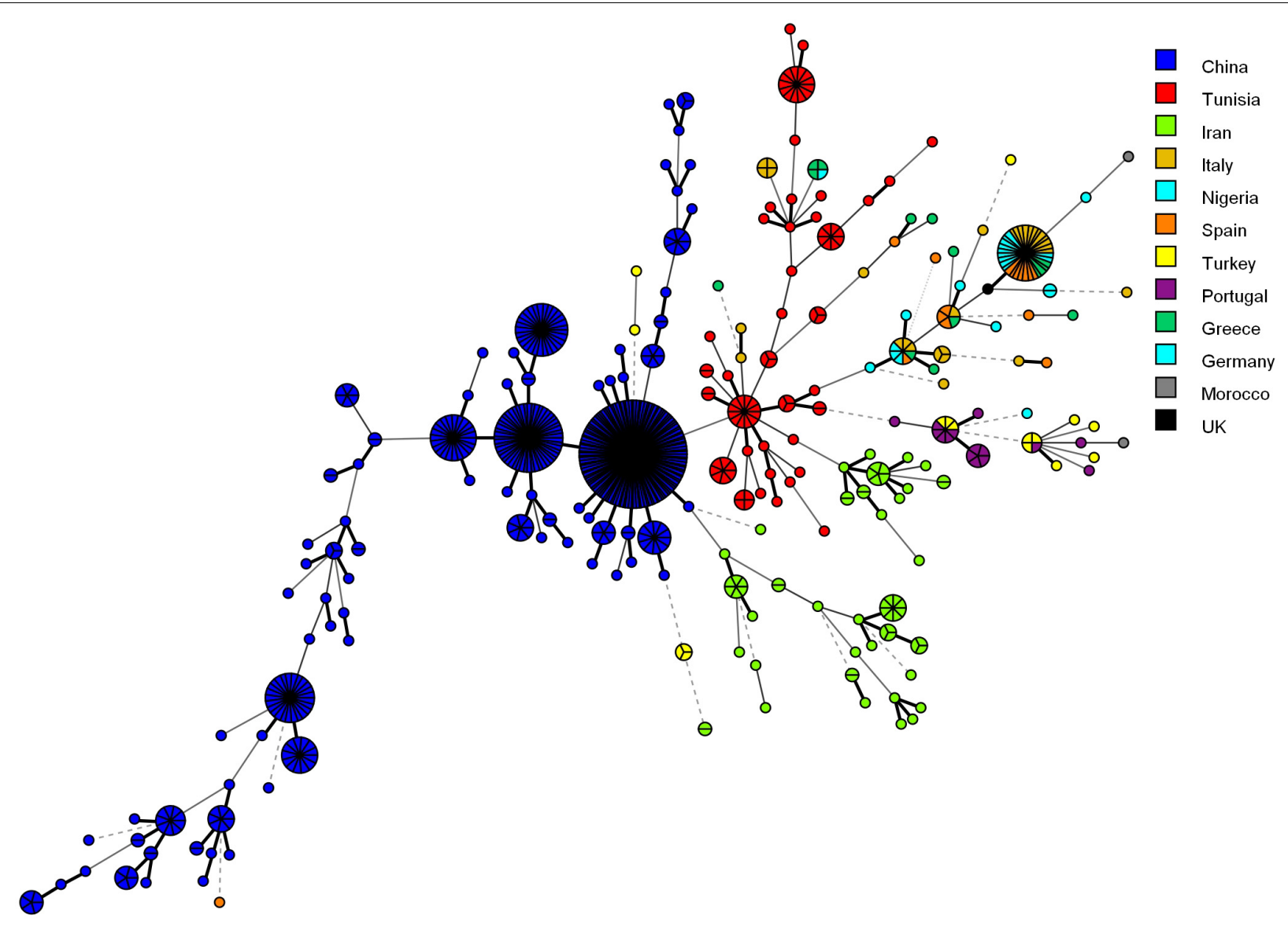

FIGURE 4 | Minimum spanning tree indicating the genotypic diversity of $C$. glabrata isolates from the southwest of Iran compared to those of other countries. Each one of the colored circles indicates one country. Isolates from Iran $(n=61)$, China $(n=411)$, Tunisia $(n=85)$, Italy $(n=30)$, Nigeria $(n=17)$, Spain $(n=16)$, Germany $(n=6)$, Greece $(n=15)$, Morocco $(n=2)$, Portugal $(n=15)$, and Turkey $(n=16)$. Furthermore, a standard strain (CBS2175) from the United Kingdom was used.

of 61 C. glabrata strains using six microsatellite markers. In general, although we used the same panel of six microsatellite markers in our study as that used by previous studies, our DP value (0.964) was not consistent with those of Chillemi et al. (2016) ( $\mathrm{DP}=0.89$ for 127 unrelated isolates) and Hou et al. (2017) ( $\mathrm{DP}=0.88$ for 411 unrelated isolates). However, the DP obtained in our study is comparable to that of Abbes et al. (2012) ( $D P=0.941$ for 85 unrelated isolates) and is higher than the ideal value (0.95) defined by Van Belkum et al. (2007) for genotyping. Therefore, our findings show that a panel of six microsatellite markers could be an excellent typing method for C. glabrata isolates recovered from the southwest of Iran.

In our study, the population genetic structure demonstrated a genetic homogeneity $\left(\mathrm{F}_{\mathrm{ST}}=0.0053\right)$ among $C$. glabrata isolates from two groups of VVC with single and multiple episodes. This finding was inconsistent with the results of Amouri et al. (2012) who found a genetic heterozygosity between two groups of acute and recurrent VVC for C. glabrata isolates (Fst: 0.207) as well as, showing a genetic homogeneity between $C$. albicans groups (Fst $<0.05)$. In agreement with our results, Chong et al. (2003) stated a small genetic diversity not only for C. glabrata isolates but also for other Candida species such as C. albicans, C. lusitaniae, and C. famata isolated from two groups (as above). Some researcher also suggested that the occurrence of RVVC could be multifactorial and not necessarily related to specific genotypes (Chong et al., 2003; Richter et al., 2005; Amouri et al., 2011). As was mentioned in the results, most of the detected genotypes $(68.6 \%)$ were unique. This finding reflects the high genetic diversity of C. glabrata isolates from the southwest of Iran and is comparable to the results of previous studies (Abbes et al., 2012, 2015). As is shown in Figure 4, the isolates of other countries did not have identical genotypes in all loci compared to Iranian isolates. This comparison represents a relatively high level of genetic variation of C. glabrata isolates and geographical selection (Klotz et al., 2016).

Our findings highlighted a positive association of C. glabrata population structures with the predominant genotype (GT27) and $\mathrm{r} /$ non-WT to antifungal drugs which is consistent with those of Dhieb et al. (2015). In contrast, other researchers have found no correlation between the predominant genotype or genotypes with antifungal resistance (De Meeûs et al., 2002; Dodgson et al., 2003; Abbes et al., 2011; Klotz et al., 2016; Amanloo et al., 2017). This discrepancy may be due to the differences in geographical 
locations, the treatment protocols used in different areas, selfmedication by patients, sociocultural conditions of people, and other effective factors on drug resistance.

\section{CONCLUSION}

The current research is the first molecular epidemiology study in the southwest of Iran and demonstrates the antifungal susceptibility profiles of C. glabrata in it. This study shows a wide range of the genetic diversity of C. glabrata (35 different genotypes) from VVC in the southwest of Iran. The majority of the non-wild isolates had a dominant genotype or genotypes related to this dominant genotype (clonal cluster one).

\section{DATA AVAILABILITY}

The datasets generated for this study can be found in NCBI GenBank, https://www.ncbi.nlm.nih.gov/nuccore/?term=kiasat.

\section{ETHICS STATEMENT}

This project was approved by the ethical committee of the Ahvaz Jundishapur University of Medical Sciences (Registered Code:

\section{REFERENCES}

Abbes, S., Amouri, I., Sellami, H., Neji, S., Trabelsi, H., and Ayadi, A. (2015). Genetic structure of candida glabrata isolates from hospitalized and nonhospitalized patients in Sfax-Tunisia. Symbiosis 3, 1-8.

Abbes, S., Sellami, H., Sellami, A., Hadrich, I., Amouri, I., Mahfoudh, N., et al. (2012). Candida glabrata strain relatedness by new microsatellite markers. Eur. J. Clin. Microbiol. Infect. Dis. 31, 83-91. doi: 10.1007/s10096-0111280-4

Abbes, S., Sellami, H., Sellami, A., Makni, F., Mahfoudh, N., Makni, H., et al. (2011). Microsatellite analysis and susceptibility to FCZ of Candida glabrata invasive isolates in Sfax Hospital. Tunisia. Med. Mycol. 49, 10-15. doi: 10.3109/ 13693786.2010.493561

Al-Aali, K. Y. (2015). Prevalence of vaginal candidiasis among pregnant women attending Al-Hada Military Hospital, Western Region, Taif, Saudi Arabia. Int. J. Sci. Res. 4, 1736-1743.

Amanloo, S., Shams-Ghahfarokhi, M., Ghahri, M., and Razzaghi-Abyaneh, M. (2017). Genotyping of clinical isolates of Candida glabrata from Iran by multilocus sequence typing and determination of population structure and drug resistance profile. Med. Mycol. 56, 207-215. doi: 10.1093/mmy/myx030

Amirrajab, N., Badali, H., Didehdar, M., Afsarian, M. H., Mohammadi, R., Lotfi, N., et al. (2016). In vitro activities of six antifungal drugs against Candida glabrata isolates: an emerging pathogen. Jundishapur J. Microbiol. 9:e36638. doi: $10.5812 /$ jjm.36638

Amouri, I., Sellami, H., Abbes, S., Hadrich, I., Mahfoudh, N., Makni, H., et al. (2012). Microsatellite analysis of Candida isolates from recurrent vulvovaginal candidiasis. J. Med. Microbiol. 61, 1091-1096. doi: 10.1099/jmm.0.043992-0

Amouri, I., Sellami, H., Borji, N., Abbes, S., Sellami, A., Cheikhrouhou, F., et al. (2011). Epidemiological survey of vulvovaginal candidosis in Sfax, Tunisia. Mycoses 54, e499-e505. doi: 10.1111/j.1439-0507.2010.01965.x

Bahram, A., Hamid, B., and Zohre, T. (2009). Prevalence of bacterial vaginosis and impact of genital hygiene practices in non-pregnant women in zanjan, iran. Oman Med. J. 24, 288-293. doi: 10.5001/omj.2009.58

Chillemi, V., Lo Passo, C., Van Diepeningen, A. D., Rharmitt, S., Delfino, D., Cascio, A., et al. (2016). Multilocus microsatellite analysis of European and
IR.AJUMS.REC.1396.912). Furthermore, all the patients attended midwifery clinics and vaginal sampling was a routine part of their therapy. All the patients have a file in these clinics containing their signed consent forms. In addition, verbal permission was also obtained from the patients.

\section{AUTHOR CONTRIBUTIONS}

$\mathrm{AM}$ and AR-M conceived and designed the manuscript. NK performed the experiment and drafted the manuscript. NK, AM, and AR-M data analyzed and interpreted the results.

\section{FUNDING}

This study was part of a Ph.D. thesis (NK) supported by a grant (No: OG-96139) from the Ahvaz Jundishapur University of Medical Sciences, Ahvaz, Iran.

\section{ACKNOWLEDGMENTS}

We would like to thank the Infectious and Tropical Diseases Research Center, Health Research Institute, at Ahvaz Jundishapur University of Medical Sciences for their support.

African Candida glabrata isolates. Eur. J. Clin. Microbiol. Infect. Dis. 35, 885892. doi: 10.1007/s10096-016-2610-3

Chong, P. P., Lee, Y. L., Tan, B. C., and Ng, K. P. (2003). Genetic relatedness of Candida strains isolated from women with vaginal candidiasis in Malaysia. J. Med. Microbiol. 52, 657-666. doi: 10.1099/jmm.0. 04973-0

De Meeûs, T., Renaud, F., Mouveroux, E., Reynes, J., Galeazzi, G., Mallié, M., et al. (2002). Genetic structure of Candida glabrata populations in AIDS and nonAIDS patients. J. Clin. Microbiol. 40, 2199-2206. doi: 10.1128/jcm.40.6.21992206.2002

Dhieb, C., Normand, A. C., Al-Yasiri, M., Chaker, E., El Euch, D., Vranckx, K., et al. (2015). MALDI-TOF typing highlights geographical and fluconazole resistance clusters in Candida glabrata. Med. Mycol. 53, 462-469. doi: 10.1093/mmy/ myv013

Dodgson, A. R., Pujol, C., Denning, D. W., Soll, D. R., and Fox, A. J. (2003). Multilocus sequence typing of Candida glabrata reveals geographically enriched clades. J. Clin. Microbiol. 41, 5709-5717. doi: 10.1128/jcm.41.12.5709-5717. 2003

Esmaeilzadeh, S., Omran, S. M., and Rahmani, Z. (2009). Frequency and etiology of vulvovaginal candidiasis in women referred to a gynecological center in babol, lran. Int. J. Fertil. Steril. 3, 74-77.

Espinel-Ingroff, A., Barchiesi, F., Cuenca-Estrella, M., Pfaller, M. A., Rinaldi, M., Rodriguez-Tudela, J. L., et al. (2005). International and multicenter comparison of EUCAST and CLSI M27-A2 broth microdilution methods for testing susceptibilities of Candida spp. to fluconazole, itraconazole, posaconazole, and voriconazole. J. Clin. Microbiol. 43, 3884-3889. doi: 10.1128/JCM.43.8.38843889.2005

Essel, E., Amenga-Etego, L., and Quaye, S. L. (2014). A case study of the incidence and risk factors of vaginal candidiasis in a girl's senior high school in Bolgatanga, Ghana. Int. J. Health Sci. Res. 4, 212-217.

EUCAST (2017). Method for the Determination of Broth Dilution Minimum Inhibitory Concentrations of Antifungal Agents for Yeasts. EUCAST DEFINITIVE DOCUMENT E.DEF 7.3.1. Växjö: EUCAST.

Gharaghani, M., Rezaei-Matehkolaei, A., Zarei Mahmoudabadi, A., and Keikhaei, B. (2016). The frequency, antifungal susceptibility and enzymatic profiles of 
candida species isolated from neutropenic patients. Jundishapur J. Microbiol. 9:e41446. doi: 10.5812/jjm.41446

Habibipour, R. (2016). Prevalence rate of vulvovaginal candidiasis and identification of Candida species in women in referred to Hamedan hospitals 2013-2014, west of Iran. Zahedan J. Res. Med. Sci. 18:e6250.

Hou, X., Xiao, M., Chen, S. C., Kong, F., Wang, H., Chu, Y. Z., et al. (2017). Molecular epidemiology and antifungal susceptibility of Candida glabrata in China (August 2009 to July 2014): a multi-center study. Front. Microbiol. 8:880. doi: $10.3389 /$ fmicb. 2017.00880

Jamilian, M., Mashhadi, E., Sarmadi, F., Ghaznavirad, A., Bani, J. M., Farhadi, E., et al. (2007). Frequancy of vulvovaginal Candidiasis species in nonpregnant 15-50 years old women in spring 2005 in Arak. Arak Univ. Med. Sci. J. 10, 7-14.

Klotz, U., Schmidt, D., Willinger, B., Steinmann, E., Buer, J., Rath, P. M., et al. (2016). Echinocandin resistance and population structure of invasive Candida glabrata isolates from two university hospitals in Germany and Austria. Mycoses 59, 312-318. doi: $10.1111 /$ myc. 12472

Mahmoudabadi, A. Z., Najafyan, M., and Alidadi, M. (2010). Clinical study of Candida vaginitis in Ahvaz, Iran and susceptibility of agents to topical antifungal. Pak. J. Med. Sci. 26, 607-610.

Makanjuola, O., Bongomin, F., and Fayemiwo, S. A. (2018). An update on the roles of non-albicans Candida Species in Vulvovaginitis. J. Fungi 4:121. doi: 10.3390/jof 4040121

Meletiadis, J., Curfs-Breuker, I., Meis, J. F., and Mouton, J. W. (2017). In Vitro antifungal susceptibility testing of candida isolates with the EUCAST methodology, a new method for ECOFF determination. Antimicrob. Agents Chemother. 61, e02372-16. doi: 10.1128/AAC.02372-16

Mohamadi, J., Havasian, M. R., Panahi, J., and Pakzad, I. (2015). Antifungal drug resistance pattern of Candida. spp isolated from vaginitis in IlamIran during 2013-2014. Bioinformation 11, 203-206. doi: 10.6026/973206300 11203

Mohammadi-Ghalehbin, B., Javanpour Heravi, H., Arzanlou, M., and Sarvi, M. (2016). Prevalence and antibiotic resistance pattern of Candida spp. isolated from pregnant women referred to health centers in Ardabil, Iran. J. f Ardabil Univ. Med. Sci. 16, 409-421.

Rad, M. M., Zafarghandi, S., Abbasabadi, B., and Tavallaee, M. (2011). The epidemiology of Candida species associated with vulvovaginal candidiasis in an Iranian patient population. Eur. J. Obstetr. Gynecol. Reprod. Biol. 155, 199-203. doi: 10.1016/j.ejogrb.2010.11.022

Rezaei-Matehkolaei, A., Shafiei, S., and Zarei-Mahmoudabadi, A. (2016). Isolation, molecular identification, and antifungal susceptibility profiles of vaginal isolates of Candida species. Iran J. Microbiol. 8, $410-417$.

Richter, S. S., Galask, R. P., Messer, S. A., Hollis, R. J., Diekema, D. J., and Pfaller, M. A. (2005). Antifungal susceptibilities of Candida species causing vulvovaginitis and epidemiology of recurrent cases. J. Clin. Microbiol. 43, 2155-2162. doi: 10.1128/JCM.43.5.2155-2162.2005

Sardi, J. C., Scorzoni, L., Bernardi, T., Fusco-Almeida, A. M., and Mendes Giannini, M. J. (2013). Candida species: current epidemiology, pathogenicity, biofilm formation, natural antifungal products and new therapeutic options. J. Med. Microbiol. 62, 10-24. doi: 10.1099/jmm.0.045054-0

Segal, E., and Frenkel, M. (2018). Experimental in vivo models of Candidiasis. J Fungi 4:21. doi: 10.3390/jof4010021

Shokohi, T., Bandalizadeh, Z., Hedayati, M. T., and Mayahi, S. (2011). In vitro antifungal susceptibility of Candida species isolated from oropharyngeal lesions of patients with cancer to some antifungal agents. Jundishapur J. Microbiol. 4, S19-S26.

Silva, S., Rodrigues, C. F., Araujo, D., Rodrigues, M. E., and Henriques, M. (2017). Candida species biofilms' antifungal resistance. J. Fungi 3:8. doi: 10.3390/ Jof3010008

Van Belkum, A., Tassios, P., Dijkshoorn, L., Haeggman, S., Cookson, B., Fry, N., et al. (2007). Guidelines for the validation and application of typing methods for use in bacterial epidemiology. Clin. Microbiol. Infect. 13, 1-46. doi: 10.1111/ j.1469-0691.2007.01786.x

White, T. J., Bruns, T., Lee, S., and Taylor, J. (1990). "Amplification and direct sequencing of fungal ribosomal RNA genes for phylogenetics," in PCR Protocols: a Guide to Methods and Applications, Vol. 18, eds M. A. Innis, D. H. Gelfand, J. J. Sninsky, and T. J. White (New York, NY: Academic Press), 315-322. doi: 10.1016/b978-0-12-372180-8.50042-1

Conflict of Interest Statement: The authors declare that the research was conducted in the absence of any commercial or financial relationships that could be construed as a potential conflict of interest.

Copyright (C) 2019 Kiasat, Rezaei-Matehkolaei and Mahmoudabadi. This is an openaccess article distributed under the terms of the Creative Commons Attribution License (CC BY). The use, distribution or reproduction in other forums is permitted, provided the original author(s) and the copyright owner(s) are credited and that the original publication in this journal is cited, in accordance with accepted academic practice. No use, distribution or reproduction is permitted which does not comply with these terms. 